\title{
Desafios e oportunidades de repensar o processo de trabalho em Vigilância Sanitária: um relato de experiência
}

\section{Challenges and opportunities to rethink the Health Surveillance work process: an experience report}

\author{
Milton Cosme Ribeiro,** iD \\ Sandra Oquendo Bedoya" (iD) \\ Vanessa Alves Ferreira'll'
}

Ana Maria Caldeira Oliveira'

Escola de Saúde Pública do Estado de Minas Gerais, MG, Brasil

" Faculdade de Ciências Biológicas e da Saúde, Universidade Federal dos Vales do Jequitinhonha e Mucuri, Diamantina, MG, Brasil

II' Departamento de Nutrição, Escola de Enfermagem, Universidade Federal de Minas Gerais, Belo Horizonte, MG, Brasil

\footnotetext{
* E-mail: milton.ribeiro@saude.mg.gov.br
}

\begin{abstract}
RESUMO
Introdução: Trata-se de um relato de experiência pautado no processo de aprendizagem no trabalho como ferramenta para superação de dificuldades vivenciadas por trabalhadores da área de vigilância sanitária. Objetivo: Elencar os desafios e as possibilidades para o aprimoramento das ações de vigilância sanitária, a partir da reflexão crítica do próprio processo de trabalho. Método: 0 relato foi elaborado a partir de registros e observações de debates realizados em reuniões realizadas entre os trabalhadores. Resultados: As reuniões em grupo criaram um espaço dialógico dentro do espaço cotidiano de trabalho permitindo discussões enriquecedoras sobre os processos de trabalho em vigilância sanitária. Conclusões: Os espaços de diálogos possibilitaram uma reflexão crítica do processo de trabalho e fizeram com que os trabalhadores de vigilância sanitária fossem capazes de reconhecer os desafios e propor alternativas para a sua própria atuação profissional.
\end{abstract}

PALAVRAS-CHAVE: Vigilância Sanitária; Trabalhador; Saúde; Educação

\begin{abstract}
Introduction: This is an experience report based on the learning process at work as a tool to overcome difficulties experienced by health surveillance workers. Objective: To list the challenges and possibilities for improving health surveillance actions, based on a critical reflection of the work process itself. Method: The report was prepared from records and observations of debates conducted at meetings held between workers. Results: The group meetings created a dialogical space within the daily workspace, allowing enriching discussions about the health surveillance work processes. Conclusions: The dialogue spaces enabled a critical reflection of the work process and made health surveillance workers able to recognize the challenges and propose alternatives for their own professional performance.
\end{abstract}

KEYWORDS: Health Surveillance; Worker; Health; Education 


\section{INTRODUÇ̃̃O}

As recentes transformações na forma de produção de bens e serviços no mundo contemporâneo, relacionadas ao desenvolvimento de novas tecnologias e à facilidade de acesso à informação, exigem que os profissionais estejam mais preparados para enfrentar questões cada vez mais complexas. Alguns autores relataram que o enfrentamento desses desafios perpassa pelo processo de aprendizagem no trabalho, que muitas vezes pode estar associado à experiência baseada no desenvolvimento das pessoas a partir de problemas reais, como forma de gerar aprendizado e influenciar as ações grupais e organizacionais ${ }^{1,2}$.

A problematização da realidade é importante, pois desperta no sujeito a sensação de incômodo, a percepção de que sua prática não está respondendo às necessidades de saúde das pessoas. Este incômodo só pode ser despertado quando ele é percebido, vivido de forma intensa. A vivência e a reflexão sobre as práticas de trabalho são os caminhos ideais para produzir insatisfações e disposição para elaborar alternativas que visem enfrentar os desafios ${ }^{3}$.

O trabalho da Vigilância Sanitária (Visa) apresenta especificidades devido à natureza dos objetos de intervenção e do caráter exclusivamente estatal e disciplinador de suas ações. Os seus objetos de intervenção podem ser entendidos como objetos complexos, que se situam no espaço de relações entre a ciência, a saúde e o mercado ${ }^{4}$.

Segundo Souza e Costa ${ }^{4}$, os elementos que compõem o processo de trabalho da Visa podem ser assim sistematizados: 1) objeto de trabalho - produtos, serviços, processos e ambientes de interesse da saúde; 2 ) meios de trabalho - instrumentos materiais, normas técnicas e jurídicas e saberes mobilizados para a realização do trabalho de controle sanitário; 3) agentes do trabalho - agentes do estado que atuam no aparato institucional da Visa; 4) produto do trabalho - controle dos riscos sanitários sobre produtos, serviços, processos e ambientes de interesse da saúde; e 5) finalidade do trabalho - proteção e defesa da saúde coletiva.

Dessa maneira, os trabalhadores de Visa intervêm no risco sanitário através de regulamentação, controle e fiscalização das relações de produção e consumo de bens e serviços relacionados à saúde. Tendo em vista a natureza variada desses riscos, cabe a esses profissionais a análise permanente e atenta dos fatores determinantes, bem como exige constante interação com os produtores, os prestadores de serviços e a população ${ }^{5}$.

A metodologia tradicional de ensino voltada para trabalhadores de Visa não somente limita o diálogo entre os trabalhadores, como não contribuí para a reflexão da realidade local, ou seja, não produzem mudanças significativas para melhoria das ações desenvolvidas pelo serviço em questão ${ }^{6}$. Em outras palavras, os atuais processos de qualificação na área de vigilância sanitária ainda são voltados para a realização de treinamentos descontínuos e não para uma formação dos trabalhadores. Deste modo, é preciso repensar novos modelos e estratégias de aprendizagem.
Considerando os desafios e estratégias de superação priorizados pelo Ciclo de Debates em vigilância sanitária realizado pela Agência Nacional de Vigilância Sanitária (Anvisa) em 2015, podem ser citados, entre outros: a escassez de profissionais, a sobrecarga de trabalho e a falta de uma política de capacitação permanente/continuada nos processos de trabalho para acompanhar a modernização do setor regulado e novas tecnologias; e elencadas estratégias como: definir uma política pública de recursos humanos para tratar da formação e qualificação dos fiscais com a elaboração de um programa de educação permanente e continuada sistematizado e de habilitação técnica, com planos de capacitação para os servidores e responsabilização dos três entes do Sistema Nacional de Vigilância Sanitária (SNVS) ${ }^{7}$.

Entretanto, como bem observado por Oliveira e Lanni ${ }^{8}$ o cotidiano dos trabalhadores de Visa municipais exige uma pronta ação. Exige que as propostas saiam do papel, rapidamente, objetivando garantir o acesso desses trabalhadores à qualificação. Ceccim e Feuerwerker ${ }^{9}$ observaram que serão necessários novos mecanismos de planejamento e gestão para que os serviços possam ser espaços de aprendizagem.

Nesse contexto, o objetivo do estudo foi promover a autorreflexão crítica sobre o processo de trabalho, a fim de elencar desafios e oportunidade para o enfrentamento de possíveis entraves existentes no serviço, utilizando-se das reuniões de trabalho como espaços de diálogo entre trabalhadores de Visa.

\section{MÉTODO}

Este trabalho faz parte de uma das atividades do curso de especialização em Gestão do Trabalho e Educação em Saúde (CEGTES) promovido pela Escola de Saúde Pública do Estado de Minas Gerais (ESP-MG). O curso foi ofertado em função de uma diretriz política da Secretária de Gestão do Trabalho e da Educação em Saúde e do Departamento de Regulação e Gestão do Trabalho do Ministério da Saúde, desenvolvido no âmbito do Programa de Qualificação e Estruturação da Gestão do Trabalho e da Educação no Sistema Único de Saúde (ProgeSUS), que visou qualificar as áreas de gestão do trabalho e da educação em saúde nas secretarias municipais e estaduais de saúde em âmbito nacional, em parceria com a Escola Nacional de Saúde Pública Sérgio Arouca da Fundação Oswaldo Cruz (ENSP/Fiocruz) ${ }^{10}$.

A atividade consistiu em realizar uma experiência no ambiente de trabalho para verificar como seria o resultado de uma prática pedagógica. $O$ texto traz apenas relatos narrados pelos autores sem identificação de nomes, frases, dados ou qualquer outra informação dos participantes, bem como sem qualquer exposição a riscos e danos.

A técnica aplicada baseou-se no método disposto no Manual Criativo e llustrado de Brainstorming para Comunicadores Organizacionais $^{11}$, que propõe práticas de como pensar sem limites, ideias a fio, soluções improváveis para os problemas do cotidiano e promover uma série de ideias que, recombinadas, chegam a uma resposta viável e criativa para os problemas dentro da organização. 
0 relato foi realizado a partir de registros das reuniões compostas por um grupo de técnicos de Visa que atuam em uma das 28 unidades regionais de saúde da Secretaria de Estado da Saúde de Minas Gerais. Esse órgão é responsável por realizar diversas ações em Visa, tais como atendimento a denúncias; fiscalizações; ações de informação, comunicação e educação sanitária; autorização para dispensação de medicamentos de controle especial; autorização para comercialização de retinoides; entre outras. Nenhuma dessas unidades conta com um departamento que realize atividades de educação ou formação para estes trabalhadores, que, esporadicamente passam por capacitações pontuais, segundo interesse da administração.

As reuniões de trabalho relativas a essa experiência ocorreram na própria unidade onde os mesmos exerciam suas atividades, no período de maio a setembro de 2016. No total foram realizados quatro encontros com duração de duas a quatro horas, um por mês. Todos os participantes estavam cientes dos objetivos dessa experiência. E houve concordância em participar das reuniões assim como, de posterior divulgação dos resultados.

Os participantes totalizaram seis profissionais de Visa que atuam no Núcleo de Vigilância Sanitária (NUVISA), sendo quatro deles autoridades sanitárias, responsáveis por realizar inspeções sanitárias nos estabelecimentos e serviços da região, e os outros dois responsáveis por desenvolver atividades técnicas e administrativas do setor.

Inicialmente foi escolhido um brainstormer ou facilitador entre os participantes, que seria responsável pelos registros das informações. Segundo Menezes ${ }^{12}$, o trabalho do facilitador está na aplicação de metodologias que provoquem mudanças no sentido da reflexão contextualizada voltada para as relações do e no trabalho. 0 relato foi elaborado a partir de registros e observações de debates realizados em reuniões entre os trabalhadores, tendo como premissa a reflexão crítica do próprio processo de trabalho.

Os participantes foram levados a responder perguntas relacionadas as dificuldades encontradas em sua prática profissional e em como melhorar o processo de trabalho na sua área de atuação, a partir da sua experiência. Pela técnica, todas as ideias são válidas, ou seja, nenhuma ideia é excluída e muito menos julgada, todas elas são anotadas para posterior análise até a conclusão. Os dois primeiros encontros objetivaram promover o debate acerca dos obstáculos que afetavam a qualidade do serviço prestado e os dois últimos, de problematizar e propor alternativas para enfrentamento desses obstáculos.

\section{RESULTADOS E DISCUSSÃO}

As reuniões em grupo permitiram a interação entre os participantes e promoveram discussões bastante enriquecedoras sobre os processos de trabalho em Visa. Durante os debates, os trabalhadores revelaram a existência de entraves que comprometiam diretamente a efetividade das ações de vigilância sanitária na identificação, controle e eliminação dos riscos sanitários. Entre os principais problemas levantados foram citados cinco, a seguir: 1) a priorização de atendimento às demandas espontâneas; 2) a ausência de padronização dos procedimentos de monitorização e avaliação de riscos sanitários encontrados nas inspeções; 3) o adiamento de ações diante do surgimento de outras demandas; 4) a inexistência de procedimento e fluxo de atendimento às denúncias que entram no setor; e 5) erros na apresentação de documentos para requisição de receitas de medicamentos de controle especial por secretários de saúde e médicos prescritores.

De acordo com os trabalhadores, a demanda por inspeção sanitária considera principalmente a solicitação de licenciamento por parte da empresa, sem levar em consideração o grau de risco presente em cada atividade. Para o grupo, deveriam ser priorizadas, por exemplo: atividades de maior risco como consultórios com procedimentos invasivos, farmácias de manipulação, hospitais e serviços de urgência e emergência. Como atividades de médio risco sanitário, a maioria dos trabalhadores considerou as indústrias de alimentos e os laboratórios de análises clínicas. É possivel observar que os estabelecimentos considerados de maior risco pelos trabalhadores demonstram maior complexidade tecnológica e estrutural.

Importante destacar que a Anvisa publicou recentemente, a Resolução da Diretoria Colegiada (RDC) $n^{\circ} 153$, de 26 de abril de $2017^{13}$, que dispõe sobre a classificação do grau de risco para as atividades econômicas sujeitas à vigilância sanitária. Um dos objetivos dessa resolução é, exatamente, orientar os processos de trabalho em Visa, no que se refere à priorização das atividades com base no risco sanitário. Entretanto, é necessário observar também que o risco à saúde nem sempre guarda relação direta com a complexidade das ações sanitárias, uma vez que as irregularidades com potencial de gerar agravos à saúde podem estar presentes nos objetos de baixa e alta complexidade, sujeitos à vigilância sanitária ${ }^{14}$.

Para os técnicos, a ausência de monitoramento e a avaliação de riscos sanitários favorece a baixa resolutividade sobre as situações de risco encontradas durante as fiscalizações. Eles consideram como fatores que impactam negativamente no processo de trabalho: 1) o não retorno ao estabelecimento após a primeira inspeção no período de um ano; 2) a ausência de avaliação do relatório da inspeção anterior; e 3) a alta rotatividade entre os profissionais nas inspeções realizadas em um mesmo produtor ou prestador de serviço. Outros estudos demonstraram que as dificuldades enfrentadas pelos profissionais de Visa também incluem um elevado número de tarefas, a falta de veículos de transporte e o baixo número de funcionários ${ }^{15}$.

A vivência relatada pelo grupo permitiu verificar que a ausência de uma agenda programada e de procedimentos de gerenciamento do risco sanitário são os fatores que mais impactam na efetividade das ações de vigilância sanitária e geram maior custo de postergação, embora a governabilidade para realizar essas duas ações seja alta.

Os trabalhadores também debateram o conceito de risco sanitário com base em suas experiências e conhecimentos. Na visão da maioria dos trabalhadores de Visa, o risco sanitário esteve associado à ideia de probabilidade, demonstrando o grau de incerteza em que ele se apresenta nas ações de vigilância sanitária. Neste sentido, 
para alguns autores, a diversidade de significados atribuídos a categoria "risco sanitário", pelos profissionais, relaciona-se à causa e efeito. Na maioria das vezes, está associada a um evento não desejado que poderá ou não ocorrer. Existe, portanto, elementos subjetivos envolvidos no conceito de risco que envolvem as representações e os significados que os atores sociais atribuem a essa categoria, fruto do conhecimento, seja ele formal ou não formal desses profissionais, no contexto em que eles estão inseridos ${ }^{16,17}$.

A partir de uma análise crítica do próprio processo de trabalho, os participantes apontaram a necessidade de elaboração de uma programação de inspeções sanitárias com critérios preestabelecidos, que considere não somente a demanda espontânea, mas que contribua para criação de uma rotina mais organizada e efetiva das ações de vigilância sanitária. Estes critérios poderiam considerar viagens com inspeções programadas em estabelecimentos com a licença sanitária expirada ou próxima do vencimento e a realização de inspeções a partir de uma classificação de riscos encontrados nos produtos e estabelecimentos, como forma de sistematizar a aplicação da norma.

Visando a tomada de decisão, o monitoramento, a notificação e a avaliação de riscos e situações de risco foram citados pelos trabalhadores como atividades a serem implantadas no órgão de Visa, de forma a produzir dados sobre riscos sanitários decorrentes de inspeções sanitárias. Isso possibilitaria, por exemplo, a criação de instrumentos de classificação de risco para subsidiar o planejamento das inspeções sanitárias futuras.

A criação de uma agenda para realizar pequenas reuniões de planejamento e avaliação voltadas para a análise e discussão dos relatórios de inspeção dos estabelecimentos foi apontada pelos trabalhadores como uma estratégia importante para melhoria do processo de trabalho. Essa afirmação corrobora com o estudo de Guimarães e Corvino ${ }^{6}$, realizado com técnicos municipais de Visa, que apontaram para a necessidade da construção de um espaço dentro do serviço para discutir questões relacionadas ao processo de trabalho que, na visão desses autores, poderia se constituir como uma das formas de buscar soluções viáveis aos problemas encontrados em suas rotinas.

Também foi debatida a necessidade de promover diálogos entre o órgão e os prestadores de serviços ou responsáveis por estabelecimentos produtores, a fim de melhorar a comunicação entre a Visa e o setor regulado, favorecendo a troca de informações, a consciência sanitária e a responsabilização de todos no controle e prevenção de riscos à saúde. Sob esse prisma, acreditamos que a vigilância sanitária se aproxima do conceito de promoção à saúde, fundamentalmente porque a promoção considera a participação dos sujeitos das ações na solução dos seus próprios problemas, de forma a se transformarem em sujeitos capazes de pensar criticamente e agir de modo responsável na mudança da sua realidade ${ }^{18}$.

Entre as oportunidades propostas pelos técnicos, podem ser destacadas: 1) realizar a programação das inspeções sanitárias considerando o risco sanitário; 2) notificar os riscos e situações de risco; 3) criar instrumentos para classificação de risco; 4) criar uma agenda para realização de pequenas reuniões de planejamento e avaliação das inspeções; 5) promover diálogos com prestadores de serviços e responsáveis por estabelecimentos produtores.

Deste modo, acredita-se que a criação de espaços de discussões nos serviços de Visa pode constituir uma estratégia para promover melhorias nas ações e atividades inerentes ao processo de trabalho dos profissionais.

Vale ressaltar que uma das principais dificuldades encontradas nesse estudo foi garantir a presença de todos os participantes em todas as reuniões. Isso demonstra o quanto esse tipo de prática ainda não está institucionalizada no cotidiano de trabalho em Visa.

Por fim, a experiência permitiu observar que a maioria das ações de vigilância sanitária ainda mantém características do modelo cartorial e burocrático, centrado em demandas espontâneas. Por essa razão, o estudo aponta para a necessidade de propor outras alternativas de reorganização do processo de trabalho, a partir da ótica dos próprios profissionais que atuam na execução das ações de promoção, proteção e recuperação da saúde.

\section{CONCLUSÕES}

Os resultados dessa experiência apontam que a criação de espaços de diálogos entre os trabalhadores de Visa se apresentou como uma estratégia viável na troca de conhecimentos entre os profissionais e promoveu uma reflexão crítica do processo de trabalho no âmbito local. Os trabalhadores de Visa demonstraram ser capazes de reconhecer os obstáculos para a sua atuação profissional e de propor alternativas de melhorias em suas rotinas de trabalho, com base em suas próprias experiências, criando assim oportunidades de mudanças.

Enfim, o estudo reforça a importância de novas estratégias com vistas à aprendizagem no trabalho, que constituam verdadeiras iniciativas voltadas para superação de desafios históricos no processo de construção de um sistema de vigilância sanitária mais integral e resolutivo, que considere a participação dos trabalhadores.

\section{REFERÊNCIAS}

1. Gimenez AB. Estratégias de aprendizagem no trabalho: uma prática exigida pela atualidade. Gest Soc. 2020;14(37):3306-37. https://doi.org/10.21171/ges.v14i37.2669

2. Coelho FA, Borges-Andrade JE. Uso do conceito de aprendizagem em estudos relacionados ao trabalho e organizações. Paideia. 2008;18(40):221-34. https: //doi.org/10.1590/\$0103-863X2008000200002

3. Merhy EE, Feuerwerker LCM, Ceccim RB. Educación permanente en salud: una estrategia para intervenir en la micropolítica del trabajo en salud. Salud Colectiva. 2006;2(2):147-60. https://doi.org/10.18294/sc.2006.62 
4. Souza GS, Costa EA. Considerações teóricas e conceituais acerca do trabalho em vigilância sanitária, campo específico do trabalho em saúde. Cienc Saude Coletiva. 2010;15(3):3329-40. https://doi.org/10.1590/S1413-81232010000900008

5. Rangel-s ML, Marques T, Costa EA. Risco, vigilância sanitária e comunicação: subsídios para uma política de proteção e promoção a saúde. In: Costa EA, Rangel-S ML. Comunicação em vigilância sanitária: princípios e diretrizes para uma política. Salvador: Universidade Federal da Bahia; 2007. p. 15-42.

6. Guimarães DA, Corvino MPF. Estratégias de educação permanente em saúde na percepção dos profissionais de vigilância sanitária. Vigil Sanit Debate. 2016;4(1):58-63. https://doi.org/10.3395/2317-269x.00477

7. Agência Nacional de Vigilância Sanitária - Anvisa. Desafios e estratégias de superação priorizados pelo ciclo de debates em VISA 2015. Brasília: Agência Nacional de Vigilância Sanitária; 2015.

8. Oliveira $A M C$, Ianni AMZ. Caminhos para a vigilância sanitária: o desafio da fiscalização nos serviços de saúde. Vigil Sanit Debate. 2018;6(3):4-11. https://doi.org/10.22239/2317-269x.01114

9. Ceccim RB, Feuerwerker LCM. 0 quadrilátero da formação para a área de saúde: ensino, gestão, atenção e controle social. Physis. 2004;14(1):41-65. https://doi.org/10.1590/S0103-73312004000100004

10. Escola de Saúde Pública do Estado de Minas Gerais ESP-MG. Informações: especialização em gestão do trabalho e educação em saúde - CEGTES. Belo Horizonte: Escola de Saúde Pública do Estado de Minas Gerais; 2016[acesso 22 mar 2020]. Disponível em: http://www.esp.mg.gov.br/espmg-003-de-2018/story/1410-informacoes-especializacaoem-gestao-do-trabalho-e-educacao-em-saude-cegtes

11. Juliana RC. Brainfood, dude! Manual criativo e ilustrado de brainstorming para comunicadores organizacionais [graduação]. Brasília: Universidade de Brasília; 2014[acesso 11 fev 2020]. Disponível em: https://bdm.unb.br/ bitstream/10483/9843/2/2014_JulianaRaposoCiarlini_ Manual.pdf

12. Menezes SF. Diálogos de aprendizagem com gestores da vigilância sanitária: percepções do facilitador. Vigil Sanit Debate. 2013;1(3):59-65. https://doi.org/10.3395/vd.v1i3.52

13. Agência Nacional de Vigilância Sanitária - Anvisa. Resolução $\mathrm{RDC} \mathrm{N}^{\circ} 153$, de 26 de abril de 2017. Dispõe sobre a classificação do grau de risco para as atividades econômicas sujeitas à vigilância sanitária, para fins de licenciamento, e dá outras providências. Diário Oficial União. 27 abr 2017.

14. Agência Nacional de Vigilância Sanitária - Anvisa. Categorização das ações de vigilância sanitária: minuta para discussão. Brasília: Agência Nacional de Vigilância Sanitária; 2005.

15. Santos CVB, Brandespim DF. Características dos recursos humanos e desafios no trabalho de coordenadores de vigilância em saúde no interior de Pernambuco. Vigil Sanit Debate. 2018;6(2):54-60. https://doi.org/10.22239/2317-269x.01027

16. Silva AVF, Lana FCF. Significando o risco sanitário: modos de atuação sobre o risco na vigilância sanitária. Vigil Sanit Debate. 2014;2(2):17-26. https://doi.org/10.3395/vd.v2i2.145

17. Freitas FP, Santos BMO. Irregularidades sanitárias como marcador de risco à saúde: um desafio para a vigilância sanitária. Vigil Sanit Debate. 2013;1(1):43-51. https://doi.org/10.3395/vd.v1i1.4

18. Chagas MF, Villela WV. Vigilância sanitária e promoção de saúde: apontamentos para além da regulação e controle. Vigil Sanit Debate. 2014;2(3):30-6. https://doi.org/10.3395/vd.v2i3.178

\section{Contribuição dos Autores}

Ribeiro MC, Bedoya AO - Concepção, planejamento (desenho do estudo), aquisição, análise e interpretação dos dados e redação do trabalho. Ferreira VA - Análise e interpretação dos dados e redação do trabalho. Oliveira AMC - Concepção, planejamento (desenho do estudo) e redação do trabalho. Todos os autores aprovaram a versão final do trabalho.

Os autores informam não haver qualquer potencial conflito de interesse com pares e instituições, políticos ou financeiros deste estudo.

Esta publicação está sob a licença Creative Commons Atribuição 3.0 não Adaptada.

Para ver uma cópia desta licença, visite http://creativecommons.org/licenses/by/3.0/deed.pt_BR. 